\title{
Identification of suitable reference genes for gene expression studies using quantitative polymerase chain reaction in lung cancer in vitro
}

\author{
HASSAN ALI ${ }^{1,2}$, ZHENWU DU ${ }^{1,3}$, XIUYING LI ${ }^{4}$, QIWEI YANG ${ }^{4}$, \\ YU CHENG ZHANG ${ }^{4}$, MEI WU ${ }^{4}$, YI $\mathrm{LI}^{2}$ and GUIZHEN ZHANG ${ }^{1,3}$ \\ ${ }^{1}$ Department of Central Laboratory, Second Hospital, Jilin University, Changchun, Jilin 130041; \\ ${ }^{2}$ Department of Immunology, Norman Bethune College of Medicine, Jilin University, Changchun, Jilin 130021; \\ ${ }^{3}$ Institute of Orthopedics, Second Hospital, Jilin University, Changchun, Jilin 130041; \\ ${ }^{4}$ Department of Central Research, China-Japan Hospital, Jilin University, \\ Changchun, Jilin 130033, P.R. China
}

Received January 15, 2014; Accepted June 5, 2014

DOI: $10.3892 / \mathrm{mmr} .2015 .3159$

\begin{abstract}
The present study aimed to examine 10 housekeeping genes (HKGs), including 18s ribosomal RNA (18S), glyceraldehyde-3-phosphate dehydrogenase $(G A P D H)$, ribosomal protein large $\mathrm{P0}(R P L P O), \beta$-actin $(A C T B)$, peptidylprolyl isomerase A (PPIA), phosphoglycerate kinase-1 $(P G K 1), \beta$-2-microglobulin $(B 2 M)$, ribosomal protein LI3a (RPL13A), hypoxanthine phosphoribosyl transferase-1 (HPRT1) and TATA box binding protein $(T B P)$ in order to identify the most stable and suitable reference genes for use in expression studies in non-small cell lung cancer. The mRNA expression encoding the panel of the 10 HKGs was determined using reverse transcription-quantitative PCR (RT-qPCR) in human lung cancer cell lines. Three software programs, BestKeeper, NormFinder and geNorm, were used to ascertain the most suitable reference genes to normalize the RNA input. The present study examined three lung cancer cell lines (A549, NCI-H446 and NCI-H460). The analysis of the experimental data using BestKeeper software revealed that all 10 HKGs were stable, with $G A D P H$, followed by $18 \mathrm{~S}$ being the most stable genes and PPIA and HPRT1 being the least stable genes. The NormFinder software results demonstrated that PPIA followed by $A C T B$ were the most stable and $B 2 M$ and $R P L P O$ were the least stable. The geNorm software results revealed that $A C T B$ and $P G K 1$, followed by $P P I A$ were the most stable genes and $B 2 M$ and $R P L P O$ were identified as the least stable genes. Due to discrepancies in the ranking orders
\end{abstract}

Correspondence to: Professor Guizhen Zhang, Department of Central Laboratory, Second Hospital, Jilin University, 9 Ziqiang Street, Changchun, Jilin 130041, P.R. China

E-mail: zhanguizhenjlu@163.com

Key words: reverse transcription-quantitative polymerase chain reaction, housekeeping genes, normalization, lung cancer of the reference genes obtained by different analyzing software programs, it was not possible to determine a single universal reference gene. The suitability of selected reference genes requires unconditional validation prior to each study. Based on the three analyzing programs, $A C T B, P P I A$ and $P G K 1$ were the most stable reference genes in lung cancer cell lines.

\section{Introduction}

Lung cancer is the most commonly diagnosed malignancy and is the leading cause of mortality among all types of cancer. Every year, lung cancer contributes to more than one million mortalities worldwide, among which non-small cell lung cancer (NSCLC) accounts for $85 \%$ of cases $(1,2)$. NSCLC can be divided into three types, including adenocarcinoma, large cell lung carcinoma and squamous cell carcinoma (3). Each of these share a common set of carcinoma characteristics. Cell lines derived from each of the main lung tumor types are widely used as experimental models in lung cancer biology (4). Reverse transcription-quantitative polymerase chain reaction (RT-qPCR) has revolutionized the field of gene expression analysis in living organisms (5). The main advantages of RT-qPCR are its superior specificity, sensitivity and broad quantification range $(6,7)$. Despite being a useful technique, there are challenges coupled with its use, an important one being the normalization with an accurate and reliable reference gene, referred to as a housekeeping gene (HKG) $(8,9)$. The term housekeeping gene was initially used to describe genes that are essential for cell function. Ideal HKGs are stably expressed in each cell type, do not respond to external stimuli and exhibit little or no run-to-run or sample-to-sample RT-qPCR variation. They are an internal reference to which target gene expression can be associated in order to correct unspecific variation caused by an imprecise amount of input RNA, RNA degradation or the presence of reaction inhibitors $(8,10)$. Reference genes are often selected from the literature and are used across several experimental conditions, some of which may enhance the differences in the expression of a reference 
Table I. Candidate reference genes and their respective symbols and functions used in the present study.

\begin{tabular}{|c|c|c|}
\hline Symbol & Name & Function \\
\hline $18 \mathrm{~S}$ & 18s ribosomal RNA & Component of the 40 s ribosomal subunit \\
\hline GAPDH & Glyceraldehyde-3-phosphate dehydrogenase & Oxidoreductase in glycolysis and gluconeogenesis \\
\hline RPLPO & Ribosomal protein large P0 & Component of the 60 s ribosomal subunit \\
\hline$A C T B$ & $\beta$-actin & Protein involved in various types of cell motility \\
\hline PPIA & $\begin{array}{l}\text { Peptidylprolyl isomerase A, } \\
\text { cydophilin A, romatase A }\end{array}$ & $\begin{array}{l}\text { Accelerates the folding of proteins, catalyzes the cis-tans } \\
\text { isomerization of proline imidic peptide bonds in } \\
\text { oligopeptides }\end{array}$ \\
\hline$P G K 1$ & Phosphoglycerate kinase-1 & Enzyme involved in glycolysis \\
\hline$B 2 M$ & $\beta$-2-microglobulin & $\begin{array}{l}\text { Component of major histocompatibility complex class } 1 \\
\text { molecules }\end{array}$ \\
\hline RPL13A & Ribosomal protein LI3a & Structural component of the large $60 \mathrm{~S}$ ribosomal subunit \\
\hline HPRT1 & Hypoxanthine phosphoribosyl transferase-1 & Enzyme involved in purine synthesis in salvage pathway \\
\hline$T B P$ & TATA box binding protein & General RNA polymerase 11 transcription factor \\
\hline
\end{tabular}

gene under certain conditions. Previous studies have indicated that certain commonly used HKGs, including $\beta$-actin $(A C T B)$ and glyceraldehyde-3-phosphate dehydrogenase $(G A P D H)$ are differentially expressed in various tissues (11-13). The reliability of normalized data is reliant on the robustness of reference genes. If unrecognized, unexpected changes in the expression of reference genes could result in flawed conclusions of real biological effects. Therefore, identification of stable and reliable reference genes is a prerequisite to any reliable analysis of RT-qPCR data. Numerous reference genes, including GADPH, ACTB, $\beta$-2-microglobulin $(B 2 M)$ and ribosomal protein large $\mathrm{P} 0$ ( $R P L P O)$ have been identified, and their suitability for gene expression studies in diverse human tissue and cell types has been validated (14-17). RT-qPCR has been used in lung cancer studies to enumerate the expression of predictive and or prognostic targets (18). In the present study, three types of lung cancer cell lines (NCI-H A549, NCI-H446 and NCI-H460) were assembled and 10 common HKGs, including 18S, GAPDH, RPLPO, ACTB, peptidylprolyl isomerase A (PPIA), phosphoglycerate kinase-1 (PGKI), B2M, ribosomal protein LI3a (RPL13A), hypoxanthine phosphoribosyl transferase-1 (HPRTI) and TATA box binding protein $(T B P)$ (Table I) were selected in order to examine their stability and suitability for RT-qPCR normalization in NSCLC using three common statistical algorithms, NormFinder, geNorm and BestKeeper. Candidate HKGs were selected on the basis of two criteria: i) their previous use as a reference gene and ii) their ability to cover a wide expression spectrum.

\section{Materials and methods}

Cell lines. Human lung cancer cell lines A549 and NCI-H446 were purchased from the American Type Culture Collection (Manassas, VA, USA) and NCI-H460 was provided by the Central Gene Therapy Department of China-Japan Union Hospital, Jilin University (Changchun, Jilin, China). Cells were cultured in RPMI-1640 medium (Gibco-BRL, Carslbad, CA, USA), supplemented with $10 \%$ fetal bovine serum (Gibco-BRL) and 100 units of penicillin (Sigma-Aldrich,
St. Louis, MO, USA), and maintained at $37^{\circ} \mathrm{C}$ in a $5 \% \mathrm{CO}_{2}$ humidified atmosphere.

RNA extraction. The cell lines A549, NCI-H446 and NCI-H460 were cultured for $72 \mathrm{~h}$, and total RNA was extracted from each cell using TRIzol reagent (Takara Bio, Inc., Shiga, Japan) according to the manufacturer's instructions. Briefly, $1 \mathrm{ml}$ of TRIzol reagent was used to homogenize the cells $\left(\sim 2 \times 10^{6}\right)$. Samples were thoroughly mixed and incubated at room temperature for $5 \mathrm{~min}$. The samples were then treated with $0.2 \mathrm{ml}$ chloroform (Haodeng Industrial Co., Ltd, Shanghai, China) by reverse mixing. Phase separation was performed by placing the samples at room temperature for $5 \mathrm{~min}$ followed by centrifugation at $12,000 \mathrm{xg}$ and $4^{\circ} \mathrm{C}$ for $15 \mathrm{~min}$. The aqueous layer was mixed with $0.5 \mathrm{ml}$ isopropanol (Haodeng Industrial Co., Ltd) to precipitate the RNA. Samples were placed at room temperature for $10 \mathrm{~min}$ and centrifuged at $12,000 \mathrm{x} \mathrm{g}$ and $4^{\circ} \mathrm{C}$ for $10 \mathrm{~min}$. The RNA pellet was washed with $1 \mathrm{ml} 75 \%$ alcohol and centrifuged at $10,000 \mathrm{xg}$ and $4^{\circ} \mathrm{C}$ for $5 \mathrm{~min}$. The pellet was air dried and resuspended with DNA/RNAase free water. The purity and concentration of RNA was determined using NanoDrop 1000 (Thermo Scientific, Waltham, MA, USA) spectrophotometry.

Complementary DNA (cDNA) synthesis. Total RNA $(1 \mu \mathrm{g})$ from each cell group was reverse-transcribed to cDNA using a First Strand cDNA Synthesis kit (GeneCopoeia, Guangzhou, China) according to the manufacturer's instructions. The cDNA was stored at $-20^{\circ} \mathrm{C}$.

Quantitative PCR. For RT-qPCR analysis, SYBR Green Premix EX Taq (Takara Bio, Inc.) was used in a reaction mixture that comprised 5 pmol of each gene-specific primer and $40 \mathrm{ng}$ of cDNA sample, in a final volume of $20 \mu \mathrm{l}$. The primer sequences used (Table II) were synthesized by Sangon Biotech Co., Ltd. (Shanghai, China). RT-qPCR was performed using an AB Prism 7500 PCR detection system (Applied Biosystems, Foster City, CA, USA), under the following conditions: $30 \mathrm{sec}$ of polymerase activation at $95^{\circ} \mathrm{C}$ followed 
Table II. Primer sequences, product sizes and PCR efficiencies of candidate reference genes.

\begin{tabular}{lllcc}
\hline Symbol & \multicolumn{1}{c}{ Forward primer } & \multicolumn{1}{c}{ Reverse primer } & $\begin{array}{c}\text { Product size } \\
\text { (bp) }\end{array}$ & $\begin{array}{c}\text { PCR } \\
\text { efficiency }\end{array}$ \\
\hline $18 \mathrm{~S}$ & GTGGAGCGATTTGTCTGGTT & AACGCCACTTGTCCCTCTAA & 115 & 1.90 \\
GAPDH & ATGGGGAAGGTGAAGGTCG & GGGGTCATTGATGGCAACAATA & 108 & 1.99 \\
$R P L P 0$ & CTGGAAGTCCAACTACTTCC & CATCATGGTGTTCTTGCCCAT & 160 & 2.74 \\
$A C T B$ & GAAGATCAAGATCATTGCTCCT & TACTCCTGCTTGCTGATCCA & 111 & 1.89 \\
$P P I A$ & TCCTGGCATCTTGTCCAT & TGCTGGTCTTGCCATTCCT & 179 & 2.10 \\
$P G K 1$ & GCCACTTGCTGTGCCAAATG & CCCAGGAAGGACTTTACCTT & 102 & 2.62 \\
$B 2 M$ & CTATCCAGCGTACTCCAAAG & GAAAGACCAGTCCTTGCTGA & 188 & 2.08 \\
$R P L 13 A$ & CGAGGTTGGCTGGAAGTACC & CTTCTCGGCCTGTTTCCGAG & 121 & 2.00 \\
$H P R T 1$ & CCTGGCGTCGTGATTAGTGAT & AGACGTTCAGTCCTGTCCATAA & 131 & 1.78 \\
$T B P$ & GCACAGGAGCCAAGAGTGA & GTTGGTGGGTGAGCACAAG & 174 & 2.10 \\
\hline
\end{tabular}

PCR, polymerase chain reaction; GADPH, glyceraldehyde3-phosphate dehydrogenase; $R P L P 0$, ribosomal protein large $\mathrm{P} 0$; $A C T B, \beta$-actin; PPIA, peptidylprolylisomerase A; PGK1, phosphoglycerate kinase-1; B2M, $\beta-2$ microglobulin; RPL13A, ribosomal protein LI3a; HPRT1, hypoxanthine phosphoribosyl transferase-1; TBP, TATA box binding protein.

by 40 cycles of denaturation at $95^{\circ} \mathrm{C}$ for $5 \mathrm{sec}$, annealing at $58^{\circ} \mathrm{C}$ for $60 \mathrm{sec}$ and elongation at $72^{\circ} \mathrm{C}$ for $30 \mathrm{sec}$. Each assay was performed three times. The RT-PCR products were then subjected to $1 \%$ agarose gel electrophoresis containing ethidium bromide.

Statistical analysis. Data analysis was performed using ABI 7500 SDS system software (version 1.4; Applied Biosystems). All biological replicates were used to calculate the average threshold cycle $(\mathrm{Ct})$ values. The stability of the 10 candidate reference genes was comprehensively evaluated using NormFinder (version 0953; http://moma.dk/normfinder-software) (16) algorithms, geNorm (version 3.4; http://medgen. ugent.be/ jvdesomp/genorm/) (19) and BestKeeper (version 1; http://www.gene-quantification.com/bestkeeper.html) $(20,21)$. In order to enter the $\mathrm{Ct}$ values into geNorm and NormFinder software, the $(\mathrm{Ct})$ values were converted into relative quantities using the following formula: $2^{-\Delta \mathrm{Ct}}(\Delta \mathrm{Ct}=\mathrm{Ct}$-lowest $\mathrm{Ct})$. The raw data was entered into the BestKeeper program and RT-qPCR efficiency was determined for each primer pair using slope analysis with a linear regression model. Relative standard curves for transcripts were performed with serial dilutions of cDNA at 1/2.5, 1/5, 1/10 and 1/20 ng. The corresponding RT-qPCR efficiencies (E) were calculated according to the following equation: $\mathrm{E}=2-{ }^{1 / \text { slope }}$.

\section{Results}

RNA purity and concentration. All RNA samples were examined for their purity and concentration. The absorbance ratio at 260/280 nm was $1.85-1.95$ for each RNA sample group, reflecting high purity and concentration.

RT-qPCR efficiency of each primer pair. The RT-qPCR efficiency of each primer pair was determined by serial dilution. The results demonstrated that the efficiencies of the HKGs of interest ranged between 1.78 (HPRTI) and 2.74 (RPLPO) for each primer pair (Table II).

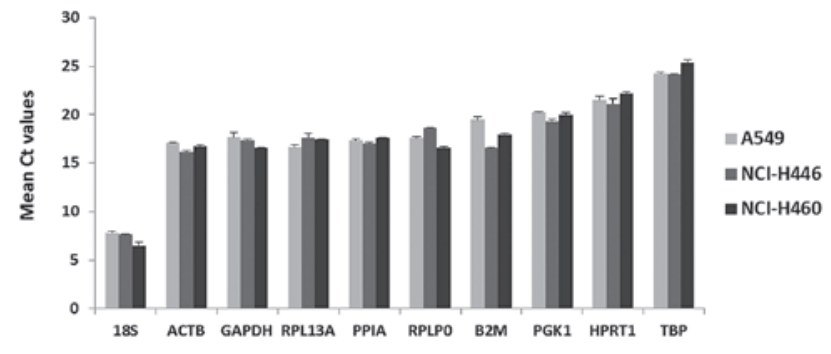

Figure 1. Mean $\mathrm{Ct}$ values of the candidate reference genes among experimental samples. Ct, threshold cycle; $A C T B, \beta$-actin; $G A D P H$, glyceraldehyde-3-phosphate dehydrogenase; $R P L 13 A$, ribosomal protein LI3a; $P P I A$, peptidylprolyl isomerase $\mathrm{A} ; R P L P 0$, ribosomal protein large $\mathrm{P} 0 ; B 2 M$, ß-2-microglobulin; $P G K 1$, phosphoglycerate kinase-1; HPRT1, hypoxanthine phosphoribosyl transferase-1; TBP, TATA box binding protein.

Candidate reference gene expression levels and ranges. In general, the 10 candidate reference genes revealed wide expression levels with mean $\mathrm{Ct}$ values in a range that is usually covered by HKGs, varying between $17.5(A C T B)$ and $25.5(T B P)$ among the three groups of lung cancer cell lines. $18 \mathrm{~S}$ revealed the lowest variability of $\mathrm{Ct}$ among all groups of the three cell lines (Fig. 1). The dissociation curve of each target reference gene demonstrated one single peak, which confirmed the specific amplification of the target reference gene (Fig. 2A). The gel electrophoresis results demonstrated one single band which further confirmed the specific RT-qPCR amplification of the target reference gene (Fig. 2B).

Candidate HKG expression stability. The expression stability of each of the 10 reference genes was analyzed using three commonly used software programs, geNorm, NormFinder and BestKeeper.

geNorm analysis. The geNorm software program is an Excel based program that calculates and compares the gene expression stability measure (M) of all candidate genes, and excludes genes with an $\mathrm{M}$-value $>1.5$. The lower the $\mathrm{M}$ value the higher 


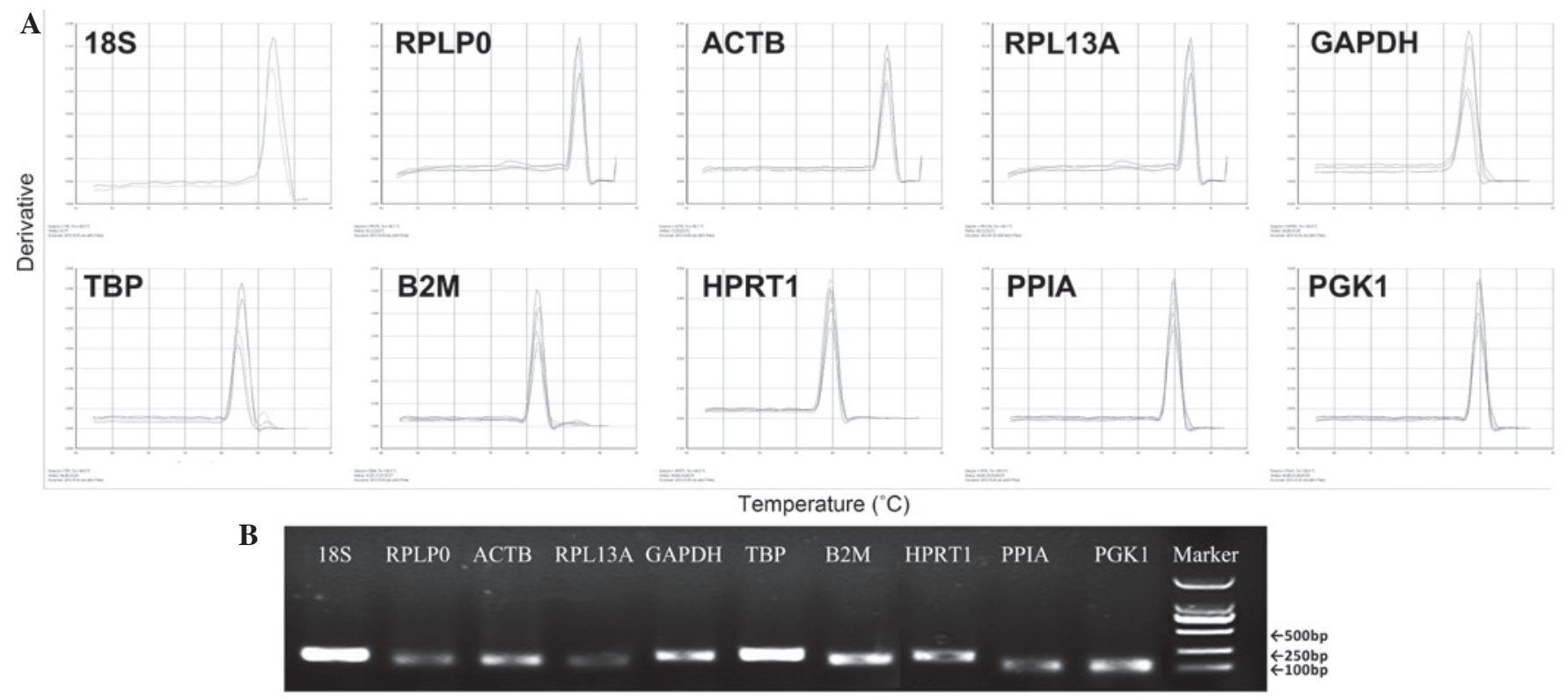

Figure 2. Reverse transcription-quantitative polymerase chain reaction product of each of the 10 target reference genes. (A) Dissociation curves of each of the 10 target reference genes. (B) $1 \%$ agarose gel electrophoresis of amplified fragments. Bp, base pairs; ACTB, $\beta$-actin; GADPH, glyceraldehyde-3-phosphate dehydrogenase; RPL13A, ribosomal protein LI3a; PPIA, peptidylprolyl isomerase A; RPLPO, ribosomal protein large P0; B2M, $\beta$-2-microglobulin; PGK1, phosphoglycerate kinase-1; HPRT1, hypoxanthine phosphoribosyl transferase-1; TBP, TATA box binding protein .

$\mathbf{A}$

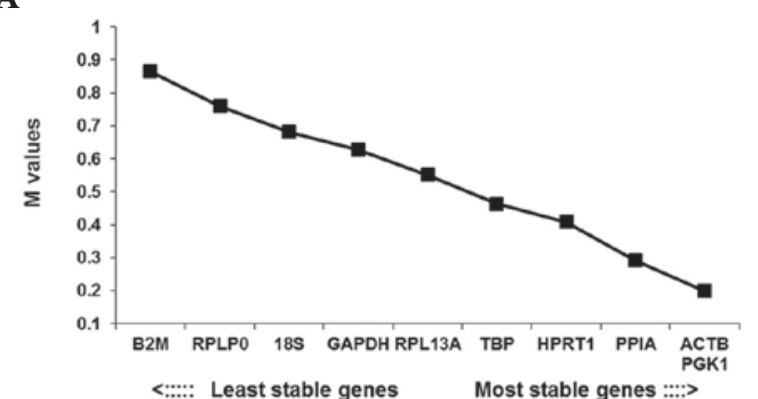

B

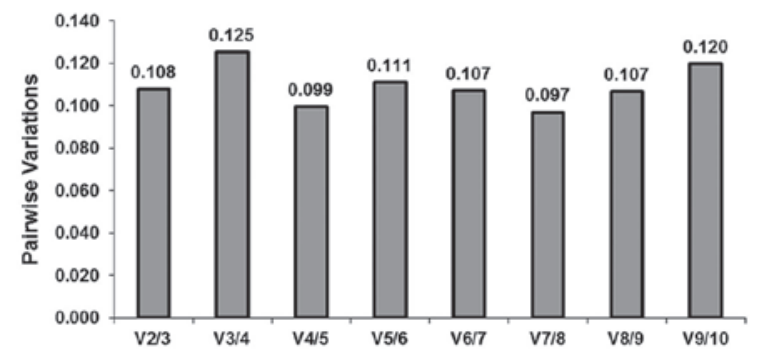

Figure 3. Stability values of the candidate control genes analyzed by geNorm. (A) Determination of the optimal number of control genes. The software calculates the normalization factor from the least two stable genes at which the variable defines the pairwise variation between two sequential normalization factors. (B) $\mathrm{M}$ value of the 10 candidate reference genes analyzed by geNorm software. The $\mathrm{x}$-axis from left to right indicates the ranking of the genes according to their stability; lower $\mathrm{M}$ values indicate higher stability. $\mathrm{M}$, gene expression stability measure; V, variation; $A C T B, \beta$-actin; $G A D P H$, glyceraldehyde-3-phosphate dehydrogenase; $R P L 13 A$, ribosomal protein LI3a; $P P I A$, peptidylprolyl isomerase A; RPLP0, ribosomal protein large $\mathrm{P} 0 ; B 2 M$, $\beta$-2-microglobulin; $P G K 1$, phosphoglycerate kinase-1; HPRT1, hypoxanthine phosphoribosyl transferase-1; TBP, TATA box binding protein.

the gene expression stability and repeats the calculations until there are two genes remaining. The $\mathrm{M}$ value indicates the average pairwise variation of a gene compared with all the

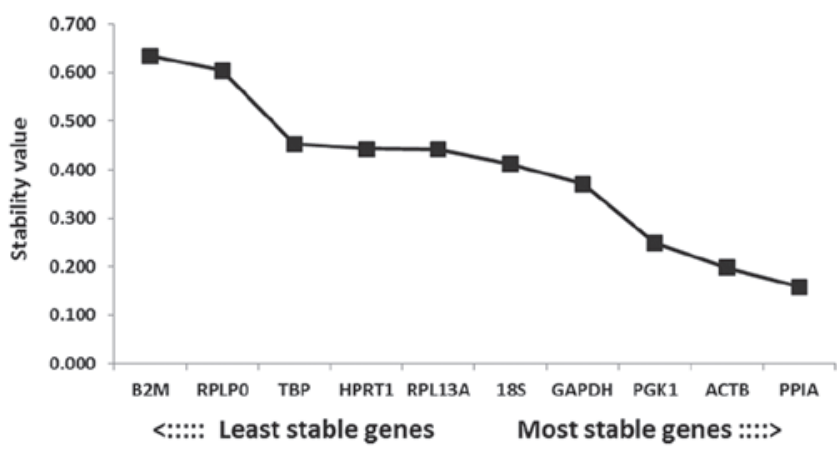

Figure 4. Stability values of the candidate reference genes calculated by NormFinder software. The lower stability values indicate higher stability. $A C T B, \beta$-actin; GADPH, glyceraldehyde-3-phosphate dehydrogenase; RPL13A, ribosomal protein LI3a; PPIA. peptidylprolyl isomerase A; RPLPO, ribosomal protein large $\mathrm{P} 0 ; B 2 M, \beta$-2-microglobulin; $P G K 1$, phosphoglycerate kinase-1; HPRT1, hypoxanthine phosphoribosyl transferase-1; TBP, TATA box binding protein.

other candidate genes. In order to determine the maximum number of genes necessary for adequate normalization in each panel of the experiment, geNorm determines pairwise variation $(\mathrm{V}) \mathrm{Vn} / \mathrm{Vn}+1 . \mathrm{V}=0.15$ which is used as a cut-off value. A value $<0.15$ indicates the number of control genes that is sufficient for valid normalization (Fig. 3A). The results demonstrated that the M-value for each of the 10 reference genes was $<1.5$, thus there was no exclusion. The most stable genes were $A C T B$ and $P G K 1$, followed by $P P I A$, while the gene with the least expression stability was $B 2 M$ followed by RPLPO (Fig. 3A).

NormFinder analysis. The NormFinder software calculates the stability value based on an estimation of intra and intergroup variation for the analyzed genes. A low stability value has a low gene expression variance and indicates high stability expression. 
Table III. Descriptive statistical analysis of candidate reference genes analyzed by BestKeeper software.

\begin{tabular}{lcccccccccc}
\hline & $18 S$ & ACTB & GAPDH & RPL13A & PPIA & RPLP0 & B2M & PGK1 & HPRT1 & TBP \\
\hline N=12 & 12 & 12 & 12 & 12 & 12 & 12 & 12 & 12 & 12 \\
Geo mean (CP) & 7.29 & 16.65 & 17.18 & 17.22 & 17.31 & 17.58 & 17.96 & 19.81 & 21.60 & 24.56 \\
Ar mean (CP) & 7.32 & 16.65 & 17.19 & 17.23 & 17.31 & 17.60 & 18.00 & 19.82 & 21.61 & 24.57 \\
Min (CP) & 6.24 & 16.06 & 16.48 & 16.39 & 17.01 & 16.50 & 16.50 & 19.04 & 20.80 & 24.07 \\
Max (CP) & 7.98 & 17.14 & 18.14 & 18.24 & 17.70 & 18.63 & 19.75 & 20.29 & 22.34 & 25.73 \\
SD ( \pm CP) & 0.55 & 0.35 & 0.41 & 0.38 & 0.21 & 0.69 & 0.99 & 0.38 & 0.53 & 0.52 \\
CV (\% CP) & 7.49 & 2.09 & 2.38 & 2.23 & 1.22 & 3.94 & 5.53 & 1.94 & 2.44 & 2.13 \\
Min (x-fold) & -2.07 & -1.50 & -1.62 & -1.78 & -1.23 & -2.12 & -2.74 & -1.71 & -1.74 & -1.41 \\
Max (x-fold) & 1.60 & 1.41 & 1.95 & 2.03 & 1.31 & 2.07 & 3.47 & 1.39 & 1.67 & 2.25 \\
SD ( \pm X-fold) & 1.46 & 1.27 & 1.33 & 1.3 & 1.16 & 1.62 & 1.99 & 1.30 & 1.44 & 1.44 \\
Coeff. of corr. (r) & 0.73 & 0.64 & 0.76 & -0.51 & 0.10 & 0.14 & 0.72 & 0.62 & -0.05 & -0.49 \\
\hline
\end{tabular}

${ }^{\text {a }} \mathrm{SD}>1.00$, was excluded from further analysis. CP, crossing point; $\mathrm{SD}$, standard deviation; $\mathrm{CV}$, coefficient of variance; ACTB, $\beta$-actin; $\mathrm{GADPH}$, glyceraldehyde3-phosphate dehydrogenase; RPL13A, ribosomal protein LI3a; PPIA, peptidylprolyl isomerase A; RPLP0, ribosomal protein large P0; B2M, $\beta$-2 microglobulin; PGK1, phosphoglycerate kinase-1; HPRT1, hypoxanthine phospho-ribosyl transferase-1; TBP, TATA box binding protein.

A
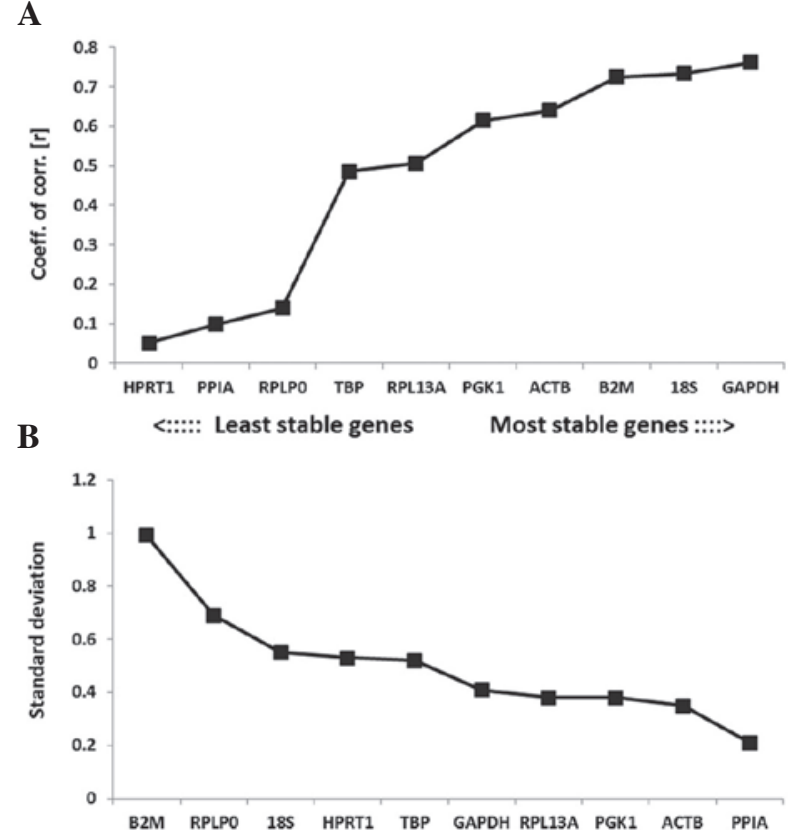

Figure 5. BestKeeper results for candidate reference genes. (A) SD was plotted on the $y$-axis. Genes with an SD $>1.00$ were excluded from further analysis. (B) Coefficient of correlation was plotted on the y-axis. A higher coefficient of correlation indicates a more stably expressed gene. SD, standard deviation; $A C T B, \beta$-actin; $G A D P H$, glyceraldehyde-3-phosphate dehydrogenase; $R P L 13 A$, ribosomal protein LI3a; PPIA, peptidylprolyl isomerase A; RPLPO, ribosomal protein large $\mathrm{P} 0 ; B 2 M, \beta$-2-microglobulin; $P G K 1$, phosphoglycerate kinase-1; HPRT1, hypoxanthine phosphoribosyl transferase-1; TBP, TATA box binding protein.

The output of this software analysis revealed that the most stable gene was PPIA followed by $A C T B$ and $P G K 1$. The least stable gene expression was $B 2 M$ followed by $R P L P O$ (Fig. 4). These results were consistent with the geNorm analysis output.

BestKeeper analysis. BestKeeper is an excel based tool that assesses the stability of candidate HKGs based on
Table IV. Ranking of candidate control genes using BestKeeper, NormFinder and geNorm software programs.

\begin{tabular}{|c|c|c|}
\hline geNorm & NormFinder & BestKeeper \\
\hline \multirow[t]{2}{*}{$A C T B, P G K 1$} & PPIA & $G A P D H$ \\
\hline & $A C T B$ & $18 \mathrm{~S}$ \\
\hline PPIA & $P G K 1$ & $B 2 M$ \\
\hline HPRT1 & $G A P D H$ & $A C T B$ \\
\hline$T B P$ & $18 \mathrm{~S}$ & $P G K 1$ \\
\hline RPL13A & RPL13A & RPL13A \\
\hline GAPDH & HPRT1 & $T B P$ \\
\hline $18 \mathrm{~S}$ & $T B P$ & RPLPO \\
\hline$R P L P O$ & $R P L P O$ & PPIA \\
\hline$B 2 M$ & $B 2 M$ & HPRT1 \\
\hline
\end{tabular}

$A C T B, \beta$-actin; $G A D P H$, glyceraldehyde-3-phosphate dehydrogenase; $R P L 13 A$, ribosomal protein LI3a; PPIA, peptidylprolyl isomerase A; $R P L P 0$, ribosomal protein large $\mathrm{P} 0 ; B 2 M, \beta$-2-microglobulin; $P G K 1$, phosphoglycerate kinase-1; HPRT1, hypoxanthine phosphoribosyl transferase-1; TBP, TATA box binding protein.

the inspection of calculated variation, including the standard deviation (SD) (22) and the coefficient of variance values (Table III). According to the BestKeeper program, the lowest variations revealed the highest stability. Genes with an SD $>1$ are considered to have an unacceptable range of variation (Fig. 5A). The analysis demonstrated that all 10 candidate HKGs had an $\mathrm{SD} \leq 1$. GAPDH was the most stable, followed by $18 \mathrm{~S}$. HPRT1 was the least stable, followed by PPIA (Fig. 5B). The results from the BestKeeper software were therefore inconsistent with those of the geNorm and NormFinder software. A summary of the rankings produced by each of the three software programs is exhibited in Table IV. 


\section{Discussion}

Lung cancer is the most common type of cancer and the most common cause of cancer-related mortality worldwide (23). NSCLC is a highly fatal disease with a poor prognosis and low survival rate (24). To increase the survival rate of patients with NSCLC, the disease must be diagnosed as early as possible. Lung tumor cell lines have been widely dispersed to and used in experimental studies, including DNA sequencing (25), microRNA and microarray analyses $(26,27)$ and detection of genome-wide methylated sequences $(28,29)$. Previous RT-qPCR has been demonstrated to be useful for early NSCLC diagnosis, prognosis, prediction and gene expression analysis (30). The use of RT-qPCR technology to study gene expression levels requires reliable normalization of data to avoid unspecific variability caused by the differences in cDNA quantity and/or quality, incorrect interpretation of experimental results and mistaken analyses. Although diverse methods are employed to normalize RT-qPCR, it remains one of the main challenges in the efficacy of this technique (31). The identification of internal control gene(s) is therefore essential for accurate quantification of target mRNA by RT-qPCR in a given set of experimental samples (32). Statistical software, including NormFinder, BestKeeper and geNorm has been developed to identify the stability of reference genes in a given set of biological samples. Several studies have used these software programs in the assessment of diverse HKGs to ascertain their suitability as reference genes for normalization of qPCR data $(17,33)$. The present study examined the RNA transcription levels of 10 common housekeeping genes, including 18S, GAPDH, RPLPO, ACTB, PPIA, PGK1, B2M, RPL13A, HPRT1 and TBP (Table I) in the NSCLC cell lines NCI-H A549, NCI-H446 and NCI-H460. The three statistical softwares NormFinder, BestKeeper and geNorm (34) were used to assess the expression level stabilities of candidate reference genes. These programs use different calculation algorithms and therefore may provide different results $(35,36)$. The present study demonstrated the following i) the purity and concentration of total RNA extracted from the abovementioned cell lines using TRIzol reagent; ii) the expression levels of the 10 reference genes determined in the above cell lines using qPCR and iii) the expression stability of the candidate reference genes in the above cell lines using geNorm, NormFinder and BestKeeper programs. In general, the present study demonstrated that almost all 10 candidate reference genes analyzed by the three independent programs could be used for future studies using lung cancer cell lines. This finding was somewhat in concordance with a previous study by Jacob et al (34). The analysis result of NormFinder was consistent with geNorm analysis output; both identified that $A C T B, P G K 1$ and PPIA were the most stable reference genes. By contrast, $B 2 M$ and $R P L P O$ were the least stable. BestKeeper analysis revealed that $G A P D H, 18 \mathrm{~S}$ and $B 2 M$ were the most stable and RPLPO, PPIA and HPRTI were the least stable reference genes. This was consistent with previous studies demonstrating that GADPH and $18 \mathrm{~S}$ were the most stable reference genes in NSCLC $(31,37)$. By contrast, another previous study using lung tissue samples demonstrated that GADPH and HPRTI were the least stable reference genes (38). Variations obtained from these three programs were expected given their distinct statistical algorithms. NormFinder and geNorm use relative quantities transformed from $\mathrm{Ct}$ values for stability calculation whereas BestKeeper uses $\mathrm{Ct}$ values directly, which may explain the different outputs from these three software programs (38). Several previous studies on reference gene selection for lung cancer also identified discrepancies between these programs $(20,37)$ and there was no agreement regarding which was the best method. Few experimental studies have analyzed the stability of potential reference genes in lung cancer cell lines. To the best of our knowledge, no previous study has analyzed 10 reference genes in lung cancer cell lines using three different statistical software programs. The present study concluded that $A C T B, P P I A$ and PGK1 were the most stable reference genes analyzed by the three statistical programs geNorm, NormFinder and BestKeeper. These findings were somewhat inconsistent with those of previous studies and it was not possible to determine a single universal reference gene. Therefore, it is suggested that appropriate reference genes require selection on the basis of specific requirements and study conditions and in consideration of the characteristics of target genes in practical applications.

\section{Acknowledgements}

This study was supported in part by grants from the Scientific Research Foundation of Jilin Province (nos. 20100942, 20110740 and 2013727038YY) and a grant from the Scientific Research Foundation of Jilin Province Development and Reform Commission (no. 2013c014-4).

\section{References}

1. Koutsokera A, Kiagia M, Saif MW, Souliotis K and Syrigos KN: Nutrition habits, physical activity and lung cancer: an authoritative review. Clin Lung Cancer 14: 342-350, 2013.

2. Perroud MW, Jr, Honma HN, Barbeiro AS, et al: Mature autologous dendritic cell vaccines in advanced non-small cell lung cancer: a phase I pilot study. J Exp Clin Cancer Res: 30: $65,2011$.

3. Travis WD: Pathology of lung cancer. Clin Chest Med 32: 669-692, 2011.

4. Watanabe T, Miura T, Degawa Y, et al: Comparison of lung cancer cell lines representing four histopathological subtypes with gene expression profiling using quantitative real-time PCR. Cancer Cell Int 10: 2, 2010.

5. Guenin S, Mauriat M, Pelloux J, Van Wuytswinkel O, Bellini C and Gutierrez L: Normalization of qRT-PCR data: the necessity of adopting a systematic, experimental conditions-specific, validation of references. J Exp Bot 60: 487-493, 2009.

6. Dheda K, Huggett JF, Bustin SA, Johnson MA, Rook G and Zumla A: Validation of housekeeping genes for normalizing RNA expression in real-time PCR. Biotechniques 37: 112-114, 2004.

7. Hendriks-Balk MC, Michel MC and Alewijnse AE: Pitfalls in the normalization of real-time polymerase chain reaction data. Basic Res Cardiol 102: 195-197, 2007.

8. Eisenberg E and Levanon EY: Human housekeeping genes, revisited. Trends Genet 29: 569-574, 2013.

9. Arenas-Hernandez M and Vega-Sanchez R: Housekeeping gene expression stability in reproductive tissues after mitogen stimulation. BMC Res Notes 6: 285, 2013.

10. Huggett J, Dheda K, Bustin S and Zumla A: Real-time RT-PCR normalisation; strategies and considerations. Genes Immun 6: 279-284, 2005.

11. Barber RD, Harmer DW, Coleman RA and Clark BJ: GAPDH as a housekeeping gene: analysis of GAPDH mRNA expression in a panel of 72 human tissues. Physiol Genomics 21: 389-395, 2005 . 
12. Bas A, Forsberg G, Hammarstrom S and Hammarstrom ML: Utility of the housekeeping genes $18 \mathrm{~S}$ rRNA, beta-actin and glyceraldehyde-3-phosphate-dehydrogenase for normalization in real-time quantitative reverse transcriptase-polymerase chain reaction analysis of gene expression in human $\mathrm{T}$ lymphocytes. Scand J Immun 59: 566-573, 2004.

13. Selvey S, Thompson EW, Matthaei K, Lea RA, Irving MG and Griffiths LR: Beta-actin - an unsuitable internal control for RT-PCR. Mol Cell Probes 15: 307-311, 2001.

14. Andersen CL, Jensen JL and Orntoft TF: Normalization of real-time quantitative reverse transcription-PCR data: a model-based variance estimation approach to identify genes suited for normalization, applied to bladder and colon cancer data sets. Can Res 64: 5245-5250, 2004.

15. Rodriguez-Mulero S and Montanya E: Selection of a suitable internal control gene for expression studies in pancreatic islet grafts. Transplantation 80: 650-652, 2005.

16. Sorby LA, Andersen SN, Bukholm IR and Jacobsen MB: Evaluation of suitable reference genes for normalization of real-time reverse transcription PCR analysis in colon cancer. J Exp Clinical Cancer Res 29: 144, 2010.

17. Chua SL, See Too WC, Khoo BY and Few LL: UBC and YWHAZ as suitable reference genes for accurate normalisation of gene expression using MCF7, HCT116 and HepG2 cell lines. Cytotechnology 63: 645-654, 2011.

18. Kim B, Lee HJ, Choi HY, et al: Clinical validity of the lung cancer biomarkers identified by bioinformatics analysis of public expression data. Cancer Res 67: 7431-7438, 2007.

19. Schlotter YM, Veenhof EZ, Brinkhof B, et al: A GeNorm algorithm-based selection of reference genes for quantitative real-time PCR in skin biopsies of healthy dogs and dogs with atopic dermatitis. Vet Immunol Immunopathol 129: 115-118, 2009.

20. Wang Q, Ishikawa T, Michiue T, Zhu BL, Guan DW and Maeda H: Stability of endogenous reference genes in postmortem human brains for normalization of quantitative real-time PCR data: comprehensive evaluation using geNorm, NormFinder and BestKeeper. Int J Legal Med 126: 943-952, 2012.

21. Pfaffl MW, Tichopad A, Prgomet $\mathrm{C}$ and Neuvians TP: Determination of stable housekeeping genes, differentially regulated target genes and sample integrity: BestKeeper - Excel-based tool using pair-wise correlations. Biotechno Lett 26: 509-515, 2004.

22. de Oliveira LA, Breton MC, Bastolla FM, et al: Reference genes for the normalization of gene expression in eucalyptus species. Plant Cell Physiol 53: 405-422, 2012.

23. Bunn PA, Jr: Worldwide overview of the current status of lung cancer diagnosis and treatment. Arch Pathology Lab Med 136: $1478-1481,2012$
24. Tsuboi M, Ohira T, Saji H, et al: The present status of postoperative adjuvant chemotherapy for completely resected non-small cell lung cancer. Ann Thoracic Cardiovasc Surg 13: 73-77, 2007.

25. Liu J, Lee W, Jiang Z, et al: Genome and transcriptome sequencing of lung cancers reveal diverse mutational and splicing events. Genome Res 22: 2315-2327, 2012.

26. Sokilde R, Kaczkowski B, Podolska A, et al: Global microRNA analysis of the NCI-60 cancer cell panel. Mol Cancer The 10: 375-384, 2011.

27. Che CL, Zhang YM, Zhang HH, et al: DNA microarray reveals different pathways responding to paclitaxel and docetaxel in non-small cell lung cancer cell line. Int J Clin Exp Path 6: 1538-1548, 2013.

28. Gazdar AF, Girard L, Lockwood WW, Lam WL and Minna JD: Lung cancer cell lines as tools for biomedical discovery and research. J Natl Cancer Inst 102: 1310-1321, 2010.

29. Gazdar AF, Gao B and Minna JD: Lung cancer cell lines: Useless artifacts or invaluable tools for medical science? Lung Cancer 68: 309-318, 2010.

30. van Eijk R, Licht J, Schrumpf M, et al: Rapid KRAS, EGFR, $\mathrm{BRAF}$ and PIK3CA mutation analysis of fine needle aspirates from non-small-cell lung cancer using allele-specific qPCR. PloS One 6: e17791,2011.

31. Saviozzi S, Cordero F, Lo Iacono M, Novello S, Scagliotti GV and Calogero RA: Selection of suitable reference genes for accurate normalization of gene expression profile studies in non-small cell lung cancer. BMC Cancer 6: 200,2006.

32. Ho-Pun-Cheung A, Bascoul-Mollevi C, Assenat E, et al: Validation of an appropriate reference gene for normalization of reverse transcription-quantitative polymerase chain reaction data from rectal cancer biopsies. Anal Biochem 388: 348-350, 2009.

33. Ohl F, Jung M, Xu C, et al: Gene expression studies in prostate cancer tissue: which reference gene should be selected for normalization? J Mol Medicine 83: 1014-1024, 2005.

34. Jacob F, Guertler R, Naim S, et al: Careful selection of reference genes is required for reliable performance of RT-qPCR in human normal and cancer cell lines. PloS One 8: e59180, 2013.

35. Mafra V, Kubo KS, Alves-Ferreira M, et al: Reference genes for accurate transcript normalization in citrus genotypes under different experimental conditions. PloS One 7: e31263, 2012.

36. Mallona I, Lischewski S, Weiss J, Hause B and Egea-Cortines M Validation of reference genes for quantitative real-time PCR during leaf and flower development in Petunia hybrida. BMC Plant Biol 10: 4, 2010.

37. Liu DW, Chen ST and Liu HP: Choice of endogenous control for gene expression in nonsmall cell lung cancer. Eur Respir J 26: 1002-1008, 2005.

38. Gresner P, Gromadzinska J and Wasowicz W: Reference genes for gene expression studies on non-small cell lung cancer. Acta Biochim Pol 56: 307-316, 2009. 